\title{
Stability of Almost Periodic Solution for a General Class of Discontinuous Neural Networks with Mixed Time-Varying Delays
}

\author{
Yingwei Li \\ College of Information Science and Engineering, Yanshan University, Qinhuangdao 066004, China \\ Correspondence should be addressed to Yingwei Li; lyw@ysu.edu.cn
}

Received 2 September 2013; Accepted 5 November 2013

Academic Editor: Huaiqin Wu

Copyright (C) 2013 Yingwei Li. This is an open access article distributed under the Creative Commons Attribution License, which permits unrestricted use, distribution, and reproduction in any medium, provided the original work is properly cited.

\begin{abstract}
The global exponential stability issues are considered for almost periodic solution of the neural networks with mixed time-varying delays and discontinuous neuron activations. Some sufficient conditions for the existence, uniqueness, and global exponential stability of almost periodic solution are achieved in terms of certain linear matrix inequalities (LMIs), by applying differential inclusions theory, matrix inequality analysis technique, and generalized Lyapunov functional approach. In addition, the existence and asymptotically almost periodic behavior of the solution of the neural networks are also investigated under the framework of the solution in the sense of Filippov. Two simulation examples are given to illustrate the validity of the theoretical results.
\end{abstract}

\section{Introduction}

When applying neural networks to solve many practical problems in optimization, control, and signal processing, neural networks are usually designed to be globally asymptotical or exponentially stable to avoid spurious responses or the problems of local minima. Hence exploring the global stability of the neural networks is of primary importance. In recent years, neural networks with discontinuous activations, as a special class of dynamical systems described by differential equations with discontinuous right-hand sides, have been found useful to address a number of interesting engineering tasks, such as dry friction, impacting machines, switching in electronic circuits, systems oscillating under the effect of an earthquake, and control synthesis of uncertain systems, and therefore have received extensive attention from a lot of scholars so far; see, for example, [1-29], and references therein. In [1], Forti and Nistri firstly dealt with the global asymptotic stability (GAS) and global convergence in finite time of a unique equilibrium point for the neural networks modeled by differential equations with discontinuous right-hand sides, and by using Lyapunov diagonally stable (LDS) matrix and constructing suitable Lyapunov function, several stability conditions were derived. In the subsequent literature, considerable efforts have been devoted to investigate the issue of stability for the neural network systems with discontinuous activation functions. In $[2,3]$, by applying generalized Lyapunov approach and $M$-matrix, Forti et al. discussed the global exponential stability (GES) of the neural networks with discontinuous or non-Lipschitz activation functions. Arguing as in [1,4], $\mathrm{Lu}$ and Chen dealt with GES and GAS of Cohen-Grossberg neural networks with discontinuous activation functions. In [5-9], under the framework of Filippov solutions, by using differential inclusion and Lyapunov approach, a series of results had been obtained for the global stability of the unique equilibrium point of the neural networks with a single constant time-delay and discontinuous activations. In [10], by using differential inclusion and constructing Lyapunov functional, Liu et al. discussed the global stability of the unique equilibrium point for the neural networks with mixed time-varying delays and discontinuous neuron activations, and some stability results were given in terms of certain LMIs. In [11], Nie and Cao studied the existence and global stability of equilibrium point for time-varying delayed competitive 
neural networks with discontinuous activation functions. In $[12,13]$, based on Lyapunov stability theory and matrix inequality analysis techniques, the authors analyzed the global robust stability of a unique equilibrium point for the neural networks with time-varying delays and discontinuous neuron activation functions. In [14], Liu and Cao discussed the robust state estimation problems for time-varying delayed neural networks with discontinuous activation functions via differential inclusions, and some criteria had been established to guarantee the existence of robust state estimator. In [15], under the framework of Filippov solutions, by using matrix measure approach, Liu et al. investigated the global dissipativity and quasisynchronization issues for the time-varying delayed neural networks with discontinuous activations and parameter mismatches.

It is well known that any equilibrium point can be regarded as a special case of periodic solution for a neuron system with arbitrary period or zero amplitude. Through the study on periodic solution, more general results can be obtained than those of the study on equilibrium point for a neuron system. Hence to study the global stability of the equilibrium point of the neural networks with discontinuous activation functions, much attention has been paid to deal with the stability of periodic solution for various neural network systems with discontinuous activations; see, for example, [17-28]. In [18-21], under the Filippov inclusion framework, by using Leray-Schauder alternative theorem and Lyapunov approach, the authors presented the conditions of the existence and GES or GAS of a unique periodic solution for Hopfield neural networks or BAM neural networks with discontinuous activation functions. In [22, 23], $\mathrm{Wu}$ et al. discussed the existence and GES of the unique periodic solution for the neural networks with discontinuous activation functions under impulsive control. In [25, 26], under the framework of Filippov solutions, by using Lyapunov approach and $H$-matrix, the authors presented the stability results of periodic solution for delayed CohenGrossberg neural networks with a single constant timedelay and discontinuous activation functions. In [27, 28], the authors explored the periodic dynamical behavior of the neural networks with time-varying delays and discontinuous activation functions. Some conditions were proposed to ensure the existence and GES of the unique periodic solution.

It should be noted that all the results reported in the literature above are concerned with the issue of stability analysis of equilibrium point or periodic solution and neglect the effects of almost periodicity for the neural networks with discontinuous activation functions. As far as we know, the almost periodicity is one of the basic properties for dynamical neural systems and appears to retrace their paths through phase space but not exactly. Almost periodic functions, with a superior spatial structure, can be regarded as a generalization of periodic functions. As pointed out by [29, $30]$, in practice, almost periodic phenomenon was more common than periodic phenomenon, and almost periodic oscillatory behavior was more accordant with reality. In the past few years, a number of results had been obtained for the global stability of the almost periodic solution of various neural networks with continuous activation functions; see, for instance, [31-39] and references therein. In [31], Ding and Wang presented the results of the existence and stability of $2^{N}$ almost periodic attractors for Cohen-Grossberg-type BAM neural networks with variable coefficients. In [37], Wang et al. investigated the multistability of delayed neural networks and described new attraction basins of almost-periodic solutions. Very recently, under the framework of the theory of Filippov differential inclusions, in [29], Allegretto et al. proved the common asymptotic behavior of almost periodic solution for discontinuous, delayed, and impulsive neural networks. In [30, 40], Lu and Chen and Qin et al. discussed the existence and uniqueness of an almost periodic solution (as well as its global exponential stability) of delayed neural networks with almost periodic coefficients and discontinuous activations. It should be pointed out that, the network model explored in $[29,30,40]$ was a class of discontinuous neural networks with a single constant time-delay, and the stability conditions were achieved by using LDS matrix or $M$-matrix. Compared with the stability conditions expressed in terms of LMIs, it was obvious that the results obtained in $[29,30,40]$ were very conservative. To the authors' knowledge, very few works have been conducted to deal with the stability analysis issues for almost periodic solution of discontinuous neural networks with time-varying delays, particularly to study the global stability of almost periodic solution by applying LMIs analysis technique, which motivates the work of this paper.

In this paper, our aim is to study the delay-dependent exponential stability problem for almost periodic solution of the neural networks with mixed time-varying delays and discontinuous activation functions. Under the framework of Filippov differential inclusions, by applying the nonsmooth Lyapunov stability theory and highly efficient LMI approach, new delay-dependent sufficient conditions are presented to ensure the existence and GES of almost periodic solution in terms of LMIs, which can be solved efficiently by using recently developed convex optimization algorithms [41]. Moreover, the obtained conclusion is applied to prove the existence and stability of periodic solution (or equilibrium point) for the neural networks with mixed time-varying delays and discontinuous activations.

For convenience, some notations are introduced as follows. $\mathbb{R}$ denotes the set of real numbers, $\mathbb{R}^{n}$ denotes the $n$ dimensional euclidean space, and $\mathbb{R}^{m \times n}$ denotes the set of all $m \times n$ real matrices. For any matrix $A, A>0(A<0)$ means that $A$ is positive definite (negative definite). $A^{-1}$ denotes the inverse of $A . A^{T}$ denotes the transpose of $A \cdot \lambda_{\max }(A)$ and $\lambda_{\text {min }}(A)$ denote the maximum and minimum eigenvalue of $A$, respectively. Given the vectors $x=\left(x_{1}, \ldots, x_{n}\right)^{T}, y=$ $\left(y_{1}, \ldots, y_{n}\right)^{T} \in \mathbb{R}^{n},\|x\|=\left(\sum_{i=1}^{n} x_{i}^{2}\right)^{1 / 2}$, and $x^{T} y=\sum_{i=1}^{n} x_{i} y_{i}$. $\|A\|$ denotes the 2 -norm of $A$; that is, $\|A\|=\sqrt{\lambda\left(A^{T} A\right)}$, where $\lambda\left(A^{T} A\right)$ denotes the spectral radius of $A^{T} A$. For $r>0$, $C\left([-r, 0] ; \mathbb{R}^{n}\right)$ denotes the family of continuous function $\varphi$ from $[-r, 0]$ to $\mathbb{R}^{n}$ with the norm $\|\varphi\|=\sup _{-r \leq s \leq 0}|\varphi(s)| . \dot{x}(t)$ denotes the derivative of $x(t)$.

Given a set $C \subset \mathbb{R}^{n}, K[C]$ denotes the closure of the convex hull of $C$ and $P_{k c}(C)$ denotes the collection of all nonempty, closed, and convex subsets of $C$. 
Let $V: \mathbb{R}^{n} \rightarrow \mathbb{R}$ be a locally Lipschitz continuous function. Clarke's generalized gradient [42] of $V$ at $x$ is defined by

$$
\begin{aligned}
\partial V(x)=K\left[\left\{\lim _{i \rightarrow \infty} \nabla V\left(x_{i}\right):\right.\right. & \\
& \left.\left.\lim _{i \rightarrow \infty} x_{i}=x, x_{i} \in \mathbb{R}^{n} \backslash \Omega_{V} \cup \mathscr{M}\right\}\right],
\end{aligned}
$$

where $\Omega_{V} \subset \mathbb{R}^{n}$ is the set of Lebesgue measure zero where $\nabla V$ does not exist and $\mathscr{M} \subset \mathbb{R}^{n}$ is an arbitrary set with measure zero.

Let $E \subset R^{n}$. A set-valued map $F: E \hookrightarrow P_{k c}\left(\mathbb{R}^{n}\right)$ is said to be measurable, if for all $y \in \mathbb{R}^{n}, \mathbb{R}^{+}$-valued function $x \rightarrow d(y, F(x))=\inf \{\|y-v\|, v \in F(x)\}$ is measurable. This definition of measurability is equivalent to saying that

$$
\operatorname{Graph}(F)=\left\{(x, v) \in E \times \mathbb{R}^{n}, v \in F(x)\right\} \in \mathscr{L}(E) \times \mathscr{B}\left(\mathbb{R}^{n}\right)
$$

(graph measurability), where $\mathscr{L}(E)$ is the Lebesgue $\sigma$-field of $E$ and $\mathscr{B}\left(\mathbb{R}^{n}\right)$ is the Borel $\sigma$-field of $\mathbb{R}^{n}$.

Let $Y, Z$ be Hausdorff topological spaces and $G(\cdot)$ : $Y \hookrightarrow 2^{Z} \backslash\{\emptyset\}$. We say that the set-valued map $G(\cdot)$ is upper semicontinuous, if, for all nonempty closed subset $C$ of $Z$, $G^{-1}(C)=\{y \in Y: G(y) \bigcap C \neq \emptyset\}$ is closed in $Y$.

The set-valued map $G(\cdot)$ is said to have a closed (convex, compact) image if, for each $x \in E, G(x)$ is closed (convex, compact).

The rest of this paper is organized as follows. In Section 2, the model formulation and some preliminaries are given. In Section 3, the existence and asymptotically almost periodic behavior of Filippov solutions are analyzed. Moreover, the proof of the existence of almost periodic solution is given. The global exponential stability is discussed, and a delaydependent criterion is established in terms of LMIs. In Section 4, two numerical examples are presented to demonstrate the validity of the proposed results. Some conclusions are drawn in Section 5.

\section{Model Description and Preliminaries}

In this paper, we consider a general class of the neural networks whose dynamics is described by the system of differential equation

$$
\begin{aligned}
\dot{x}_{i}(t)= & -d_{i} x_{i}(t)+\sum_{j=1}^{n} a_{i j} g_{j}\left(x_{j}(t)\right) \\
& +\sum_{j=1}^{n} b_{i j} g_{j}\left(x_{j}(t-\tau(t))\right)+\sum_{j=1}^{n} c_{i j} \int_{t-\sigma(t)}^{t} g_{j}\left(x_{j}(s)\right) d s \\
& +I_{i}(t), \quad i=1,2, \ldots, n
\end{aligned}
$$

or equivalently the vector form

$$
\begin{aligned}
\dot{x}(t)= & -D x(t)+A g(t)+B g(x(t-\tau(t))) \\
& +C \int_{t-\sigma(t)}^{t} g(x(x)) d s+I(t),
\end{aligned}
$$

where $x(t)=\left(x_{1}(t), x_{2}(t), \ldots, x_{n}(t)\right)^{T}$ is the vector of neuron states at time $t ; D=\operatorname{diag}\left(d_{1}, d_{2}, \ldots, d_{n}\right)$ is an $n \times n$ diagonal matrix, and $d_{i}>0, i=1, \ldots, n$, is the neuron self-inhibition; $A=\left(a_{i j}\right)_{n \times n}, B=\left(b_{i j}\right)_{n \times n}$, and $C=\left(c_{i j}\right)_{n \times n}$ are real connection weight matrices representing the weighting coefficients of the neurons; $g(x(t))=\left(g_{1}\left(x_{1}(t)\right), g_{2}\left(x_{2}(t)\right), \ldots, g_{n}\left(x_{n}(t)\right)\right)^{T}$, $g_{i}, i=1, \ldots, n$ represent the neuron input-output activations; $I(t)=\left(I_{1}\left(t_{1}\right), I_{2}\left(t_{2}\right), \ldots, I_{n}\left(t_{n}\right)\right)^{T}$ is a real vector function representing the external inputs of the neuron at time $t$; the function $\tau(t)$ and $\sigma(t)$ denote the discrete and distributed time-varying delays, respectively, satisfying

$$
\begin{array}{ll}
0 \leqslant \tau(t) \leqslant \tau_{M}, & \dot{\tau}(t) \leqslant \tau_{D}<1, \\
0 \leqslant \sigma(t) \leqslant \sigma_{M}, & \dot{\sigma}(t) \leqslant \sigma_{D}<1 .
\end{array}
$$

The activation function $g(x)$ satisfies the assumption that

$\left(A_{1}\right):(1) g_{i}, i=1, \ldots, n$, is piecewise continuous; that is, $g_{i}$ is continuous in $\mathbb{R}$ except a countable set of jump discontinuous points and, in every compact set of $\mathbb{R}$, has only a finite number of jump discontinuous points;

(2) $g_{i}, i=1,2, \ldots, n$, is nondecreasing.

Under the assumption $\left(A_{1}\right), g(x)$ is undefined at the points where $g(x)$ is discontinuous, and $K[g(x)]=$ $\left(K\left[g_{1}\left(x_{1}\right)\right], \ldots, K\left[g_{n}\left(x_{n}\right)\right]\right)^{T}$, where $K\left[g_{i}\left(x_{i}\right)\right]=\left[g_{i}\left(x_{i}^{-}\right)\right.$, $\left.g_{i}\left(x_{i}^{+}\right)\right], i=1, \ldots, n$. The system (4) is a differential equation with discontinuous right-hand side. For the system (4), we adopt the following definition of the solution in the sense of Filippov [43].

Definition 1. A function $x(t):[-l, T) \rightarrow \mathbb{R}^{n}, T \in(0,+\infty]$, is a solution of the system $(4)$ on $[-\iota, T)$ if

(1) $x(t)$ is continuous on $[-l, T)$ and absolutely continuous on $[0, T)$;

(2) $x(t)$ satisfies

$$
\begin{aligned}
\dot{x}(t) \in & -D x(t)+A K[g(x(t))]+B K[g(x(t-\tau(t)))] \\
& +C \int_{t-\sigma(t)}^{t} K[g(x(s))] d s+I(t),
\end{aligned}
$$

for a.a. $t \in[0, T)$,

where $\iota=\max \left\{\tau_{M}, \sigma_{M}\right\}$.

By the assumption $\left(A_{1}\right)(1)$, it is easy to check that $\phi$ : $(x, t) \hookrightarrow-D x(t)+A K[g(x(t))]+B K[g(x(t-\tau(t)))]+$ $C \int_{t-\sigma(t)}^{t} K[g(x(s))] d s+I(t)$ is an upper semicontinuous setvalued map with nonempty, compact, and convex values. Hence $\phi(x, t)$ is measurable [44]. By the measurable selection theorem, if $x(\cdot)$ is a solution of the system (4), then there exists a measurable function $\gamma=\left(\gamma_{1}, \gamma_{2}, \ldots, \gamma_{n}\right)^{T}:[-l, T) \rightarrow \mathbb{R}^{n}$ such that $\gamma(t) \in K[g(x(t))]$ and

$$
\begin{aligned}
\dot{x}(t)= & -D x(t)+A \gamma(t)+B \gamma(t-\tau(t)) \\
& +C \int_{t-\sigma(t)}^{t} \gamma(s) d s+I(t),
\end{aligned}
$$

for a.a. $t \in[0, T)$. 
The function $\gamma(t)$ in (7) is called an output solution associated with the state variable $x(t)$ and represents the vector of neural network outputs.

Definition 2. For any continuous function $\phi(s):[-\iota, 0] \rightarrow$ $\mathbb{R}^{n}$ and any measurable selection $\psi:[-l, 0] \rightarrow \mathbb{R}^{n}$, such that $\psi(s) \in K[g(\phi(s))]$ for a.a. $s \in[-l, 0]$, an absolutely continuous function $x(t)=x(t, \phi, \psi)$ associated with a measurable function $\gamma(t)$ is said to be a solution of the initial value problem (IVP) for the system (4) on $[0, T)$ (T might be $\infty)$ with the initial value $(\phi(s), \psi(s)), s \in[-\iota, 0]$, if

$$
\begin{gathered}
\dot{x}(t)=-D x(t)+A \gamma(t) \\
+B \gamma(t-\tau(t))+C \int_{t-\sigma(t)}^{t} \gamma(s) d s+I(t), \\
\quad \text { for a.a. } t \in[0, T), \\
x(s)=\phi(s), \quad \forall s \in[-l, 0], \\
\gamma(s)=\psi(s), \quad \text { for a.a. } s \in[-l, 0] .
\end{gathered}
$$

Definition 3 (see [45]). A continuous function $x(t): \mathbb{R} \rightarrow$ $\mathbb{R}^{n}$ is said to be almost periodic on $\mathbb{R}$, if for any scalar $\varepsilon>0$ there exist scalars $l=l(\varepsilon)>0$ and $\omega=\omega(\varepsilon)$ in any interval with the length of $l$, such that $\|x(t+\omega)-x(t)\|<\varepsilon$ for all $t \in \mathbb{R}$.

Definition 4. The almost periodic solution $x^{*}(t)$ of the system (4) is said to be globally exponentially stable, if there exist scalars $\eta>0$ and $\delta>0$, such that

$$
\left\|x(t, \phi, \psi)-x^{*}(t)\right\| \leq \eta e^{-\delta t}, \quad t \geq 0,
$$

where $x(t, \phi, \psi)$ is the solution of the system (4) with the initial value $(\phi(s), \psi(s)), s \in[-\iota, 0]$, and $\delta$ is called as the exponential convergence rate.

To obtain the main results of this paper, the following lemmas will be needed.

Lemma 5 (chain rule [42]). If $V(x): \mathbb{R}^{n} \rightarrow \mathbb{R}$ is $C$ regular and $x(t):[0,+\infty) \rightarrow \mathbb{R}^{n}$ is absolutely continuous on any compact interval of $[0,+\infty)$ then $x(t)$ and $V(x(t))$ : $[0,+\infty) \rightarrow \mathbb{R}$ are differentials for a.a. $t \in[0,+\infty)$, and

$$
\dot{V}(x(t))=\langle\varsigma, \dot{x}(t)\rangle, \quad \forall \varsigma \in \partial V(x) .
$$

Lemma 6 (Jensen's inequality). For any constant matrix $A>$ 0 , any scalars $a$ and $b$ with $b>a$, and a vector function $x(t)$ : $[a, b] \rightarrow \mathbb{R}^{n}$ such that the integrals concerned as well defined,

$$
\left(\int_{a}^{b} x(s) d s\right)^{T} A\left(\int_{a}^{b} x(s) d s\right) \leqslant(b-a) \int_{a}^{b} x^{T}(s) A x(s) d s .
$$

Lemma 7. Let scalar $v>0$. Given any $x, y \in \mathbb{R}^{n}$ and $A \in$ $\mathbb{R}^{n \times n}$,

$$
2 x^{T} A y \leqslant \frac{1}{v} x^{T} A A^{T} x+v y^{T} y .
$$

Before proceeding to the main results, we further make the following assumptions.

$\left(A_{2}\right): I_{i}(t), \tau(t)$, and $\sigma(t)$ are continuous functions and possess almost periodic property; that is, for any $\varepsilon>0$, there exist $l=l(\varepsilon)>0$ and $\omega=\omega(\varepsilon)$ in any interval with the length of $l$, such that

$$
\begin{gathered}
\left|I_{i}(t+\omega)-I_{i}(t)\right|<\varepsilon \\
|\tau(t+\omega)-\tau(t)|<\varepsilon \\
|\sigma(t+\omega)-\sigma(t)|<\varepsilon
\end{gathered}
$$

$\left(A_{3}\right)$ : For any $\eta_{i} \in K\left[g_{i}\left(x_{i}\right)\right], \zeta_{i} \in K\left[g_{i}\left(y_{i}\right)\right], \zeta_{i} \neq \eta_{i}$, there exists constant $e_{i}>0$, such that

$$
\frac{\eta_{i}-\zeta_{i}}{x_{i}-y_{i}} \leqslant e_{i}, \quad i=1,2, \ldots, n .
$$

$\left(A_{4}\right)$ : There exist a positive definite diagonal matrix $P$ and two positive definite symmetric matrices $Q, H$, such that

$$
N=\left(\begin{array}{ccc}
-P A-A^{T} P-Q-\sigma_{M} H & -P B & -P C \\
-B^{T} P & \left(1-\tau_{D}\right) Q & 0 \\
-C^{T} P & 0 & \frac{1-\sigma_{D}}{\sigma_{M}} H
\end{array}\right)
$$

$>0$.

\section{Main Results}

In this section, the main results concerned with the stability of the almost periodic solution are addressed for a general class of neural networks (4).

Firstly, we give the proof of the existence of the solution and discuss the asymptotically almost periodic behavior of the solution for the system (4).

Proposition 8. If the assumption $\left(A_{4}\right)$ holds, then there exist a positive constant $\delta<d=\min \left\{d_{1}, d_{2}, \ldots, d_{n}\right\}, a$ positive diagonal matrix $\bar{P}$, and two positive definite symmetric matrices $\bar{Q}$ and $\bar{H}$ such that

$$
R=\left(\begin{array}{cccc}
-2 D+2 \delta E_{n} & A & B & C \\
A^{T} & \Gamma & \bar{P} B & \bar{P} C \\
B^{T} & B^{T} \bar{P} & -\left(1-\tau_{D}\right) \bar{Q} & 0 \\
C^{T} & C^{T} \bar{P} & 0 & -\frac{1-\sigma_{D}}{\sigma_{M}} \bar{H}
\end{array}\right)<0
$$

where $\Gamma=\bar{P} A+A^{T} \bar{P}+e^{\delta \tau_{M}} \bar{Q}+e^{\delta \sigma_{M}} \sigma_{M} \bar{H}+\delta E_{n}$ and $E_{n}$ is the $n$-dimensional identity matrix.

Proof. Let $U=\left[\begin{array}{lll}A & B & C\end{array}\right], \lambda_{1}=\lambda_{\min }(N)$, and $\lambda_{2}=$ $\lambda_{\max }\left((1 / 2) U^{T} D^{-1} U\right)$. Choose a scalar $\theta>\lambda_{2} / \lambda_{1}$; then

$$
\theta N-\left(\frac{1}{2}\right) U^{T} D^{-1} U>0
$$

By Schur's complements, (17) is equivalent to 


$$
R_{1}=\left(\begin{array}{cccc}
-2 D & A & B & C \\
A^{T} & \theta\left(P A+A^{T} P+Q+\sigma_{M} H\right) & \theta P B & \theta P C \\
B^{T} & \theta B^{T} P & -\left(1-\tau_{D}\right) \theta Q & 0 \\
C^{T} & \theta C^{T} P & 0 & -\frac{1-\sigma_{D}}{\sigma_{M}} \theta H
\end{array}\right)<0 .
$$

Thus, we can choose a sufficiently small positive constant $\delta<$ $d$, such that

$$
\left(\begin{array}{cccc}
-2 D+2 \delta E_{n} & A & B & C \\
A^{T} & \Gamma^{\prime} & \theta P B & \theta P C \\
B^{T} & \theta B^{T} P & -\left(1-\tau_{D}\right) \theta Q & 0 \\
C^{T} & \theta C^{T} P & 0 & -\frac{1-\sigma_{D}}{\sigma_{M}} \theta H
\end{array}\right)<0,
$$

where $\Gamma^{\prime}=\theta\left(P A+A^{T} P+e^{\delta \tau_{M}} Q+e^{\delta \sigma_{M}} \sigma_{M} H\right)+\delta E_{n}$. Let $\bar{P}=\theta P$, $\bar{Q}=\theta Q$, and $\bar{H}=\theta H$; then Proposition 8 is proved.

Theorem 9. If the assumptions $\left(A_{1}\right),\left(A_{2}\right)$, and $\left(A_{4}\right)$ are satisfied, then the neural network system (4) has a solution of IVP on $[0,+\infty)$ for any initial value $(\phi(s), \psi(s)), s \in[-\iota, 0]$.

Proof. For any initial value $(\phi(s), \psi(s)), s \in[-l, 0]$, similar to the proof of Lemma 1 in [2], under the assumptions $\left(A_{1}\right)(1)$, the system (4) has a local solution $x(t)$ associated with a measurable function $\gamma(t)$ with initial value $(\phi(s), \psi(s)), s \in$ $[-l, 0]$ on $[0, T)$, where $T \in(0,+\infty)$ or $T=+\infty$ and $[0, T)$ is the maximal right-side existence interval of the local solution.

Consider the following Lyapunov functional candidate:

$$
\begin{aligned}
V(t)= & e^{\delta t} x^{T}(t) x(t)+2 \sum_{i=1}^{n} e^{\delta t} \bar{p}_{i} \int_{0}^{x_{i}(t)} g_{i}(s) d s \\
& +\int_{t-\tau(t)}^{t} e^{\delta\left(s+\tau_{M}\right)} \gamma^{T}(s) \bar{Q} \gamma(s) d s \\
& +\int_{-\sigma(t)}^{0} \int_{t+\theta}^{t} e^{\delta\left(s+\sigma_{M}\right)} \gamma^{T}(s) \bar{H} \gamma(s) d s d \theta
\end{aligned}
$$

where $\delta, \bar{P}, \bar{Q}$, and $\bar{H}$ are defined as in Proposition 8 . By Lemma 5 , by calculating the time derivative of $V(t)$ along the local solution of the system $(4)$ on $[0, T)$, it yields

$$
\begin{aligned}
& \dot{V}(t)=\delta e^{\delta t} x^{T}(t) x(t)+2 e^{\delta t} x^{T}(t) \\
& \times[-D x(t)+A \gamma(t)+B \gamma(t-\tau(t)) \\
&\left.+C \int_{t-\sigma(t)}^{t} \gamma(s) d s+I(t)\right] \\
&+2 e^{\delta t} \gamma^{T}(t) \bar{P}[-D x(t)+A \gamma(t)+B \gamma(t-\tau(t)) \\
&\left.+C \int_{t-\sigma(t)}^{t} \gamma(s) d s+I(t)\right]
\end{aligned}
$$

$$
\begin{aligned}
& +2 \delta e^{\delta t} \sum_{i=1}^{n} \bar{p}_{i} \int_{0}^{x_{i}(t)} g_{i}(s) d s+e^{\delta\left(t+\tau_{M}\right)} \gamma^{T}(t) \bar{Q} \gamma(t) \\
& -(1-\dot{\tau}(t)) e^{\delta\left(t+\tau_{M}-\tau(t)\right)} \gamma^{T}(t-\tau(t)) \bar{Q} \gamma(t-\tau(t)) \\
& +\sigma(t) e^{\delta\left(t+\sigma_{M}\right)} \gamma^{T}(t) \bar{H} \gamma(t)-(1-\dot{\sigma}(t)) \\
& \times \int_{t-\sigma(t)}^{t} e^{\delta\left(s+\sigma_{M}\right)} \gamma^{T}(s) \bar{H} \gamma(s) d s .
\end{aligned}
$$

Without loss of generality, we can suppose $0 \in K[g(0)]$. In fact, if this is not the case, set $G(x)=g(x)-\eta, \eta \in K[g(0)]$; then the system (7) could be equivalently changed as

$$
\begin{aligned}
\dot{x}(t)= & -D x(t)+A \bar{\gamma}(t)+B \bar{\gamma}(t-\tau(t)) \\
& +C \int_{t-\sigma(t)}^{t} \bar{\gamma}(s) d s+\bar{I}(t),
\end{aligned}
$$

where $\bar{\gamma}(t)=\gamma(t)-\eta \in K[G(x(t))]$, for a.a. $t \in[0, T)$, and $\bar{I}(t)=(A+B+\sigma(t) C) \eta+I(t)$. It is obvious that $0 \in K[G(0)]$. By $0<\delta<d, 0 \in K\left[g_{i}(0)\right]$, and the assumption $\left(A_{1}\right)(2)$, we have

$$
\delta \int_{0}^{x_{i}(t)} g_{i}(s) d s \leqslant \delta x_{i}(t) \gamma_{i}(t) \leqslant d_{i} x_{i}(t) \gamma_{i}(t) .
$$

Using Lemmas 6 and 7, we can obtain that

$$
\begin{aligned}
& \dot{V}(t) \leqslant e^{\delta t}\left\{2 x^{T}(t)\left(\delta E_{n}-D\right) x(t)+2 x^{T}(t) A \gamma(t)\right. \\
&+2 x^{T}(t) B \gamma(t-\tau(t)) \\
&+2 x^{T}(t) C \int_{t-\sigma(t)}^{t} \gamma(s) d s \\
&+2 \gamma^{T}(t) \bar{P} A \gamma(t)+2 \gamma^{T}(t) \bar{P} B \gamma(t-\tau(t)) \\
&+2 \gamma^{T}(t) \bar{P} C \int_{t-\sigma(t)}^{t} \gamma(s) d s+\delta \gamma^{T}(t) \gamma(t) \\
&+e^{\delta \tau_{M}} \gamma^{T}(t) \bar{Q} \gamma(t)
\end{aligned}
$$




$$
\begin{gathered}
-\left(1-\tau_{D}\right) \gamma^{T}(t-\tau(t)) \bar{Q} \gamma(t-\tau(t)) \\
+\sigma_{M} e^{\delta \sigma_{M}} \gamma^{T}(t) \bar{H} \gamma(t) \\
\left.\quad-\frac{1-\sigma_{D}}{\sigma_{M}} \int_{t-\sigma(t)}^{t} \gamma^{T}(s) d s \bar{H} \int_{t-\sigma(t)}^{t} \gamma(s) d s\right\} \\
+\frac{e^{\delta t}}{\delta}\left(I^{T}(t) I(t)+I^{T}(t) \bar{P} \bar{P} I(t)\right) \\
=e^{\delta t} z^{T} R z+\frac{e^{\delta t}}{\delta}\left(I^{T}(t) I(t)+I^{T}(t) \bar{P} \bar{P} I(t)\right) \\
\leqslant \frac{e^{\delta t}}{\delta}\left(\|I(t)\|^{2}+I^{T}(t) \bar{P} \bar{P} I(t)\right)
\end{gathered}
$$

where

$$
z=\left[\begin{array}{llll}
x^{T}(t) & \gamma^{T}(t) & \gamma^{T}(t-\tau(t)) & \int_{t-\sigma(t)}^{t} \gamma^{T}(s) d s
\end{array}\right]^{T}
$$

$R$ is defined as in Proposition 8. By the assumption $\left(A_{2}\right), I(t)$ is bounded for $t \geqslant 0$. Hence there exists a constant $M>0$ such that

$$
0<\frac{e^{\delta t}}{\delta}\left(\|I(t)\|^{2}+I^{T}(t) \bar{P} \bar{P} I(t)\right)<M, \quad t \geqslant 0 .
$$

This implies that

$$
\dot{V}(t) \leqslant M e^{\delta t}, \quad \forall t \in[0, T) .
$$

Integrating both sides of (27) from 0 to $t, t \in[0, T)$, it follows that

$$
V(t) \leqslant V(0)+\int_{0}^{t} M e^{\delta s} d s=V(0)+\frac{M}{\delta}\left(e^{\delta t}-1\right) .
$$

In view of the definition of $V(t)$ in (17) and the fact that all the terms in $V(t)$ are not negative, we have

$$
V(t) \geqslant e^{\delta t} x^{T}(t) x(t), \quad t \in[0, T) .
$$

By combining (28) and (29), it is easy to obtain

$$
\|x(t)\|^{2} \leqslant e^{-\delta t} V(0)+\frac{M}{\delta}\left(1-e^{-\delta t}\right), \quad t \in[0, T) .
$$

Therefore, $\lim _{t \rightarrow T^{-}}\|x(t)\|<+\infty$. By viability theorem in differential inclusions theory [44], one yields $T=+\infty$. That is, the system (4) has a solution of IVP on $[0,+\infty)$ for any initial value. The proof is completed.

Theorem 10. Suppose that the assumptions $\left(A_{1}\right)-\left(A_{4}\right)$ are satisfied; then any solution of the neural network system (4) is asymptotically almost periodic.
Proof. Let $x(t)$ be a solution of IVP of the system (4) associated with a measurable function $\gamma(t)$ with initial value $(\phi(s), \psi(s)), s \in[-l, 0]$. Set $y(t)=x(t+\omega)-x(t)$, we have

$$
\begin{aligned}
\dot{y}(t)= & -D x(t+\omega)+A \gamma(t+\omega)+B \gamma(t+\omega-\tau(t+\omega)) \\
& +C \int_{t+\omega-\sigma(t+\omega)}^{t+\omega} \gamma(s) d s+I(t+\omega) \\
& -[-D x(t)+A \gamma(t)+B \gamma(t-\tau(t)) \\
& \left.+C \int_{t-\sigma(t)}^{t} \gamma(s) d s+I(t)\right] \\
= & -D y(t)+A[\gamma(t+\omega)-\gamma(t)] \\
& +B[\gamma(t+\omega-\tau(t))-\gamma(t-\tau(t))]
\end{aligned}
$$$$
+C \int_{t-\sigma(t)}^{t} \gamma(s+\omega)-\gamma(s) d s+\rho(\omega, t),
$$

where

$$
\begin{aligned}
\rho(\omega, t)= & B[\gamma(t+\omega-\tau(t+\omega))-\gamma(t+\omega-\tau(t))] \\
& +C \int_{t-\sigma(t+\omega)}^{t-\sigma(t)} \gamma(s+\omega) d s+I(t+\omega)-I(t) .
\end{aligned}
$$

Consider a Lyapunov functional candidate as

$$
\begin{aligned}
W(t)= & e^{\delta t} y^{T}(t) y(t)+2 \sum_{i=1}^{n} e^{\delta t} \bar{p}_{i} \int_{0}^{y_{i}(t)} g_{i}(s) d s \\
& +\int_{t-\tau(t)}^{t} e^{\delta\left(s+\tau_{M}\right)}(\gamma(s+\omega)-\gamma(s))^{T} \\
& \times \bar{Q}(\gamma(s+\omega)-\gamma(s)) d s \\
& +\int_{-\sigma(t)}^{0} \int_{t+\theta}^{t} e^{\delta\left(s+\sigma_{M}\right)}(\gamma(s+\omega)-\gamma(s))^{T} \\
& \times \bar{H}(\gamma(s+\omega)-\gamma(s)) d s d \theta
\end{aligned}
$$

Calculating the time derivative of $W(t)$ along trajectories of the system (31), similar to the proof of Theorem 9, we can get

$$
\dot{W}(t) \leqslant \frac{e^{\delta t}}{\delta}\left(\|\rho(\omega, t)\|^{2}+\rho^{T}(\omega, t) \bar{P} \bar{P} \rho(\omega, t)\right) .
$$

From the proof of Theorem 9, we can get that $x(t)$ is bounded. Consequently, $\gamma(t)$ is also bounded. By the assumption $\left(A_{3}\right)$, there exist positive constants $\alpha$ and $\beta$, such that

$$
\begin{aligned}
\|\rho(\omega, t)\| \leqslant & \alpha\|B\||\tau(t+\omega)-\tau(t)| \\
& +\beta\|C\||\sigma(t+\omega)-\sigma(t)|+\|I(t+\omega)-I(t)\| .
\end{aligned}
$$

Therefore, by using the assumption $\left(A_{2}\right)$, it is easy to obtain that, for any $\varepsilon>0$, there exist $l=l(\varepsilon)$ and $\omega=\omega(\varepsilon)$ in any interval with the length of $l$, such that

$$
\frac{1}{\delta}\left(\|\rho(\omega, t)\|^{2}+\rho^{T}(\omega, t) \bar{P} \bar{P} \rho(\omega, t)\right) \leqslant \frac{1}{2} \delta \varepsilon^{2}, \quad t \geqslant 0 .
$$


This implies

$$
\dot{W}(t) \leqslant \frac{1}{2} \varepsilon^{2} \delta e^{\delta t} .
$$

By combining (33) and (37), we have

$$
\begin{aligned}
\|y(t)\|^{2} & \leqslant e^{-\delta t} W(t) \leqslant e^{-\delta t} W(0)+\frac{1}{2} e^{-\delta t} \int_{0}^{t} \varepsilon^{2} \delta e^{\delta s} d s \\
& =e^{-\delta t} W(0)+\frac{1}{2} \varepsilon^{2}\left(1-e^{-\delta t}\right) .
\end{aligned}
$$

Therefore, there exists $T>0$, such that, for any $t>$ $T,\|y(t)\|<(1 / \sqrt{2}) \varepsilon<\varepsilon$; that is, $\|x(t+\omega)-x(t)\|<\varepsilon$. This shows that any solution of the system (4) is asymptotically almost periodic. The proof is completed.

Remark 11. (1) If discrete time-varying delay reduces to constant delay (i.e., $\tau(t)=\tau)$, then $\rho(\omega, t)=C \int_{-\sigma(t+\omega)}^{-\sigma(t)} \gamma(s+t+$ $\omega) d s+I(t+\omega)-I(t)$. Without making the assumption $\left(A_{3}\right)$, from the proof above, it is easy to see that Theorem 10 can be also obtained. (2) If both $\tau(t)$ and $\sigma(t)$ reduce to constant delays (i.e., $\tau(t)=\tau, \sigma(t)=\sigma$ ), then $\rho(\omega, t)=I(t+\omega)-I(t)$. In this case, Theorem 10 is also true.

Next, we will discuss the existence, uniqueness, and global exponential stability of the almost periodic solutions for the system (4).

Theorem 12. Suppose that the assumptions $\left(A_{1}\right)-\left(A_{4}\right)$ hold; then the neural network system (4) has a unique almost periodic solution which is globally exponentially stable.

Proof. Firstly, we prove the existence of the almost periodic solution for the system (4).

Under the assumptions of Theorem 12, for any initial value $(\phi(s), \psi(s)), s \in[-l, 0]$, the neural network (4) has a solution which is asymptotically almost periodic. Let $x(t)$ be any solution of the system (4) associated with a measurable function $\gamma(t)$ with the initial value $(\phi(s), \psi(s)), s \in[-l, 0]$. Then

$$
\begin{aligned}
\dot{x}(t)= & -D x(t)+A \gamma(t)+B \gamma(t-\tau(t)) \\
& +C \int_{t-\sigma(t)}^{t} \gamma(s) d s+I(t),
\end{aligned}
$$

for a.a. $t \in[-l,+\infty)$.

By using (36), we can pick a sequence $\left\{t_{k}\right\}$ satisfying $\lim _{k \rightarrow+\infty} t_{k}=+\infty$ and $\left\|\rho\left(t_{k}, t\right)\right\|<1 / k$, for all $t \geqslant 0$, where $\rho(\omega, t)$ is defined in (32). In addition, the sequence $\left\{x\left(t+t_{k}\right)\right\}$ is equicontinuous and uniformly bounded. By the Arzela-Ascoli theorem and diagonal selection principle, we can select a subsequence of $\left\{t_{k}\right\}$ (still denoted by $\left\{t_{k}\right\}$ ), such that $\left\{x\left(t+t_{k}\right)\right\}$ uniformly converges into an absolute continuous function $x^{*}(t)$ on any compact set of $\mathbb{R}$.

On the other hand, since $\gamma\left(t+t_{k}\right) \in K\left[g\left(x\left(t+t_{k}\right)\right)\right]$ and $K\left[g\left(x\left(t+t_{k}\right)\right)\right]$ are bounded by the boundedness of $x(t)$, the sequence $\left\{\gamma\left(t+t_{k}\right)\right\}$ is bounded. Hence we can also select a sub-sequence of $t_{k}$ (still denoted by $\left\{t_{k}\right\}$ ), such that $\left\{\gamma\left(t+t_{k}\right)\right\}$ converges into a measurable function $\gamma^{*}(t)$ for any $t \in[-l,+\infty)$. According to the fact that

(i) $K[g(\cdot)]$ is an upper semicontinuous set-valued map,

(ii) for $t \in[-l,+\infty), x\left(t+t_{k}\right) \rightarrow x^{*}(t)$ as $k \rightarrow+\infty$,

we can get that, for any $\epsilon>0$, there exists $N>0$, such that $K\left[g\left(x\left(t+t_{k}\right)\right)\right] \subseteq K\left[g\left(x^{*}(t)\right)\right]+\epsilon \mathscr{B}$ for $k>N$ and $t \in[-l,+\infty)$, where $\mathscr{B}$ is an $n$-dimensional unit ball. Hence the fact that $\gamma\left(t+t_{k}\right) \in K\left[g\left(x\left(t+t_{k}\right)\right)\right]$ implies that $\gamma\left(t+t_{k}\right) \in$ $K\left[g\left(x^{*}(t)\right)\right]+\epsilon \mathscr{B}$. On the other hand, since $K\left[g\left(x^{*}(t)\right)\right]+\epsilon \mathscr{B}$ is a compact subset of $\mathbb{R}^{n}$, we have $\gamma^{*}(t)=\lim _{k \rightarrow+\infty} \gamma\left(t+t_{k}\right) \epsilon$ $K\left[g\left(x^{*}(t)\right)\right]+\epsilon \mathscr{B}$. Noting the arbitrariness of $\epsilon$, it follows that $\gamma^{*}(t) \in K\left[g\left(x^{*}(t)\right)\right]$ for a.a. $t \in[-l,+\infty)$. obtain

By Lebesgue's dominated convergence theorem, we can

$$
\begin{aligned}
& x^{*}(t+h)-x^{*}(t) \\
& =\lim _{k \rightarrow+\infty}\left[x\left(t+t_{k}+h\right)-x\left(t+t_{k}\right)\right] \\
& =\lim _{k \rightarrow+\infty} \int_{t}^{t+h}\left[-D x\left(t_{k}+\theta\right)+A \gamma\left(t_{k}+\theta\right)\right. \\
& +B \gamma\left(t_{k}+\theta-\tau\left(t_{k}+\theta\right)\right) \\
& \left.+C \int_{t_{k}+\theta-\sigma\left(t_{k}+\theta\right)}^{t_{k}+\theta} \gamma(s) d s+I\left(t_{k}+\theta\right)\right] d \theta \\
& =\lim _{k \rightarrow+\infty} \int_{t}^{t+h}\left[-D x\left(t_{k}+\theta\right)+A \gamma\left(t_{k}+\theta\right)\right. \\
& +B \gamma\left(t_{k}+\theta-\tau(\theta)\right) \\
& +C \int_{\theta-\sigma(\theta)}^{\theta} \gamma\left(t_{k}+s\right) d s \\
& \left.+I(\theta)+\rho\left(t_{k}, \theta\right)\right] d \theta \\
& =\int_{t}^{t+h}\left[-D x^{*}(\theta)+A \gamma^{*}(\theta)+B \gamma^{*}(\theta-\tau(\theta))\right. \\
& \left.+C \int_{\theta-\sigma(\theta)}^{\theta} \gamma^{*}(s) d s+I(\theta)\right] d \theta,
\end{aligned}
$$

for any $t \in[-l,+\infty)$ and $h \in \mathbb{R}$. This implies that $x^{*}(t)$ is a solution of the system (4).

Notice that $x(t)$ is asymptotically almost periodic; then, for any $\varepsilon>0$, there exist $T>0, l=l(\varepsilon)$, and $\omega=\omega(\varepsilon)$ in any interval with the length of $l$, such that $\|x(t+\omega)-x(t)\|<\varepsilon$, for all $t>T$. Therefore, there exists a constant $N>0$, when $k>N$ and $\left\|x\left(t+t_{k}+\omega\right)-x\left(t+t_{k}\right)\right\|<\varepsilon$, for any $t \in[-l,+\infty)$. Let $k \rightarrow+\infty$; it follows that $\left\|x^{*}(t+\omega)-x^{*}(t)\right\|<\varepsilon$, for any $t \in[-l,+\infty)$. This shows that $x^{*}(t)$ is a almost periodic solution of the system (4). 
Consider the changes of variables $z(t)=x(t)-x^{*}(t)$, which transforms (4) into the differential equation

$$
\dot{z}(t)=-D z(t)+A \widetilde{\gamma}(t)+B \tilde{\gamma}(t-\tau(t))+C \int_{t-\sigma(t)}^{t} \tilde{\gamma}(s) d s
$$

where $\widetilde{\gamma}(t) \in K[\widetilde{G}(z(t))]$ is measurable, $\widetilde{G}(z(t))=\left(\widetilde{G}_{1}\left(z_{1}(t)\right)\right.$, $\left.\widetilde{G}_{2}\left(z_{2}(t)\right), \ldots, \widetilde{G}_{n}\left(z_{n}(t)\right)\right)^{T}$ and $\widetilde{G}_{i}\left(z_{i}(t)\right)=g_{i}\left(z_{i}(t)+x_{i}^{*}(t)\right)-$ $\gamma_{i}^{*}(t)(i=1,2, \ldots, n)$ and $\gamma^{*}(t)$ is the output solution associated with $x^{*}(t)$.

Similar to $V(t)$ in (17), define a Lyapunov functional candidate $L(t)$ as

$$
\begin{aligned}
L(t)= & e^{\delta t} z^{T}(t) z(t)+2 \sum_{i=1}^{n} e^{\delta t} \bar{p}_{i} \int_{0}^{z_{i}(t)} \widetilde{G}(s) d s \\
& +\int_{t-\tau(t)}^{t} e^{\delta\left(s+\tau_{M}\right)} \tilde{\gamma}^{T}(s) \bar{Q} \widetilde{\gamma}(s) d s \\
& +\int_{-\sigma(t)}^{0} \int_{t+\theta}^{t} e^{\delta\left(s+\sigma_{M}\right)} \tilde{\gamma}^{T}(s) \bar{H} \tilde{\gamma}(s) d s d \theta .
\end{aligned}
$$

$$
\mathcal{N}=\left(\begin{array}{ccc}
-P A-A^{T} P-B^{T} B-\sigma_{M} C^{T} C & -P & -P \\
-P & \left(1-\tau_{D}\right) E_{n} & 0 \\
-P & 0 & \frac{1-\sigma_{D}}{\sigma_{M}} E_{n}
\end{array}\right)>0,
$$

then, the conclusion of Theorem 9 is still true. In fact, similar with the proof of Proposition 8 , we can choose positive constants $\theta$ and $\delta<d$, such that

$$
\mathscr{R}=\left(\begin{array}{cccc}
-2 D+2 \delta E_{n} & A & E_{n} & E_{n} \\
A^{T} & \Sigma & \theta P & \theta P \\
E_{n} & \theta P & \left(1-\tau_{D}\right) \theta E_{n} & 0 \\
E_{n} & \theta P & 0 & \frac{1-\sigma_{D}}{\sigma_{M}} \theta E_{n}
\end{array}\right)<0,
$$

where $\Sigma=\theta\left(P A+A^{T} P+e^{\delta \tau_{M}} B^{T} B+\sigma_{M} e^{\delta \sigma_{M}} C^{T} C+\delta E_{n}\right)$.

Consider the following Lyapunov functional candidate:

$$
\begin{aligned}
\mathscr{V}(t)= & e^{\delta t} x^{T}(t) x(t)+2 \theta \sum_{i=1}^{n} e^{\delta t} p_{i} \int_{0}^{x_{i}(t)} g_{i}(s) d s \\
& +\theta \int_{t-\tau(t)}^{t} e^{\delta\left(s+\tau_{M}\right)} \gamma^{T}(s) B^{T} B \gamma(s) d s \\
& +\theta \int_{-\sigma(t)}^{0} \int_{t+\rho}^{t} e^{\delta\left(s+\sigma_{M}\right)} \gamma^{T}(s) C^{T} C \gamma(s) d s d \rho .
\end{aligned}
$$

Under the assumptions $\left(A_{1}\right)$ and $\left(A_{2}\right)$, calculating the time derivative of $\mathscr{V}(t)$ along the local solution of the system (4), we can obtain

$$
\dot{\mathscr{V}}(t) \leqslant e^{\delta t} u^{T} \mathscr{R} u+\frac{e^{\delta t}}{\delta}\left(I^{T}(t) I(t)+\theta I^{T}(t) P P I(t)\right),
$$

Calculate the derivative of $L(t)$ along the solution of the system (41). Arguing as in the proof of Theorem 9, we have

$$
\dot{L}(t) \leqslant e^{\delta t} v^{T} R v-\delta e^{\delta t} z^{T}(t) z(t)-\delta e^{\delta t} \tilde{\gamma}^{T}(t) \tilde{\gamma}(t)<0,
$$

where

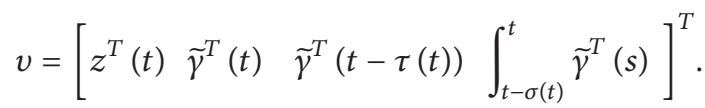

Combining (42) and (43) gives

$$
\|z(t)\| \leqslant \sqrt{L(t)} e^{-(\delta / 2) t} \leqslant \sqrt{L(0)} e^{-(\delta / 2) t} .
$$

This implies that the almost periodic solution $x^{*}(t)$ of the system (4) is globally exponentially stable. Consequently, the almost periodic solution of the system (4) is unique. The proof is completed.

Remark 13. Under the assumptions $\left(A_{1}\right)$ and $\left(A_{2}\right)$, if the assumption $\left(A_{4}\right)$ changes as the assumption $\left(\mathscr{A}_{4}\right)$, there exists a positive diagonal matrix $P$, such that the coefficient matrices $A, B$, and $C$ of the system (4) satisfy the following LMI:

where

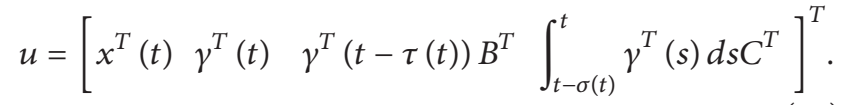

This implies that the conclusion of Theorem 9 is still true.

In addition, under the case above, if the assumption $\left(A_{3}\right)$ is satisfied, then it is easy to get that the conclusions of Theorems 10 and 12 are also correct.

Notice that periodic function can be regarded as special almost periodic function. Hence based on Theorems 9 and 12, we can obtain the following corollary.

Corollary 14. Suppose that $I(t), \tau(t)$, and $\sigma(t)$ are periodic functions; if the assumptions $\left(A_{1}\right),\left(A_{3}\right)$, and $\left(A_{4}\right)\left(\right.$ or $\left.\left(\mathscr{A}_{4}\right)\right)$ are satisfied, then

(1) the neural network system (4) has a solution of IVP on $[0,+\infty)$ for any initial value $(\phi(s), \psi(s)), s \in[-\iota, 0]$;

(2) the neural network system (4) has a unique periodic solution which is globally exponentially stable. 
When $I_{i}(t)$ is a constant external input $I_{i}$, the system (4) changes as

$$
\begin{aligned}
\dot{x}(t)= & -D x(t)+A g(t)+B g(x(t-\tau(t))) \\
& +C \int_{t-\sigma(t)}^{t} g(x(x)) d s+I .
\end{aligned}
$$

Since a constant function can be also regarded as a special almost periodic function, by applying Theorems 9 and 12 on the neural network system (51), we can obtain the following corollary.

Corollary 15. If the assumptions $\left(A_{1}\right),\left(A_{3}\right)$ and $\left(A_{4}\right)$ (or $\left.\left(\mathscr{A}_{4}\right)\right)$ are satisfied, then

(1) the neural network system (51) has a solution of IVP on $[0,+\infty)$ for any initial value $(\phi(s), \psi(s)), s \in[-l, 0]$;

(2) the neural network system (51) has a unique equilibrium point which is globally exponentially stable.

\section{Illustrative Examples}

Example 1. Consider the second-order neural network (4) with the following system parameters:

$$
\begin{gathered}
D=\left(\begin{array}{ll}
2 & 0 \\
0 & 2
\end{array}\right), \quad A=\left(\begin{array}{cc}
-3 & -0.5 \\
0.5 & -3
\end{array}\right), \\
B=\left(\begin{array}{cc}
0.85 & -0.35 \\
0.35 & 0.45
\end{array}\right), \quad C=\left(\begin{array}{cc}
0.7 & -0.45 \\
0.25 & 0.65
\end{array}\right) .
\end{gathered}
$$

Set $g_{1}(s)=[s+0.5], g_{2}(s)=[s], \tau(t)=0.8-0.2 \sin t$, and $\sigma(t)=0.5(1+\cos t)$. It is easy to check that assumptions $\left(A_{1}\right)-\left(A_{3}\right)$ hold, and $\tau_{M}=1, \tau_{D}=0.2, \sigma_{M}=1$, and $\sigma_{D}=0.5$.

Solving the LMI in (15) by using appropriate LMI solver in the Matlab, the feasible positive definite diagonal matrix $P$ and positive definite matrices $Q, H$ could be as

$$
\begin{gathered}
P=\left(\begin{array}{cc}
0.7900 & 0 \\
0 & 0.8540
\end{array}\right), \quad Q=\left(\begin{array}{cc}
1.6282 & -0.0081 \\
-0.0081 & 1.7253
\end{array}\right), \\
H=\left(\begin{array}{cc}
1.7451 & -0.0106 \\
-0.0106 & 1.8725
\end{array}\right) .
\end{gathered}
$$

The assumption $\left(A_{4}\right)$ is also satisfied.

When the external input of the network $I(t)=$ $(2 \sin t,-2 \cos t)^{T}$ is a periodic function, by Corollary 14, this neural network has a unique periodic solution which is globally exponentially stable.

Figures 1 and 2 display the state trajectories of this neural network with initial values $\phi(t)=(\sin 2 t, \cos 2 t)^{T}, t \in[-1,0]$ when $I(t)=(2 \sin t,-2 \cos t)^{T}$. It can be seen that these trajectories converge into the unique periodic solution of the network. This is in accordance with the conclusion of Corollary 14.

When the external input of the network $I(t)=(1,-3)^{T}$ is constant, by Corollary 15, this neural network has a unique equilibrium point which is globally exponentially stable.

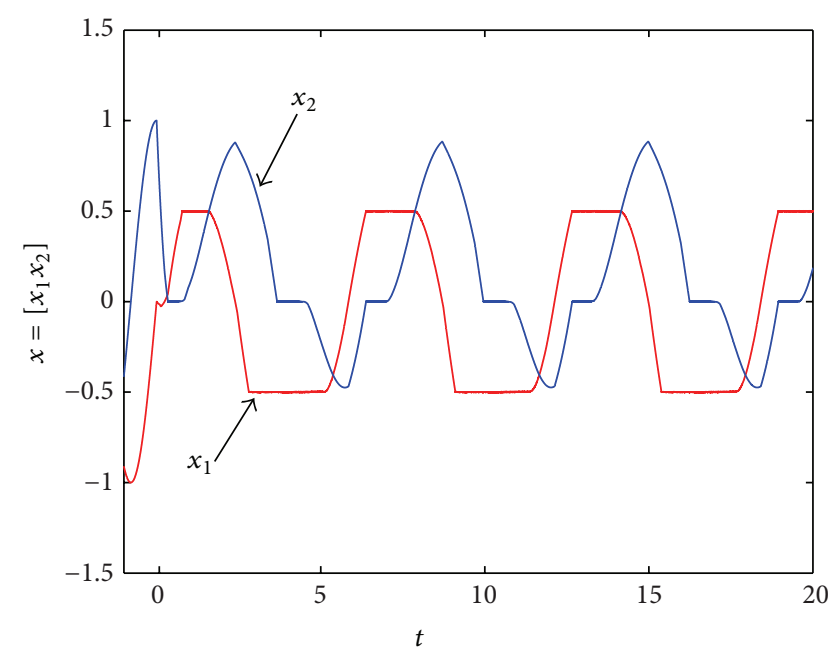

FIgURE 1: Time-domain behavior of the state variables $x_{1}$ and $x_{2}$ when $I(t)=(2 \sin t,-2 \cos t)^{T}$.

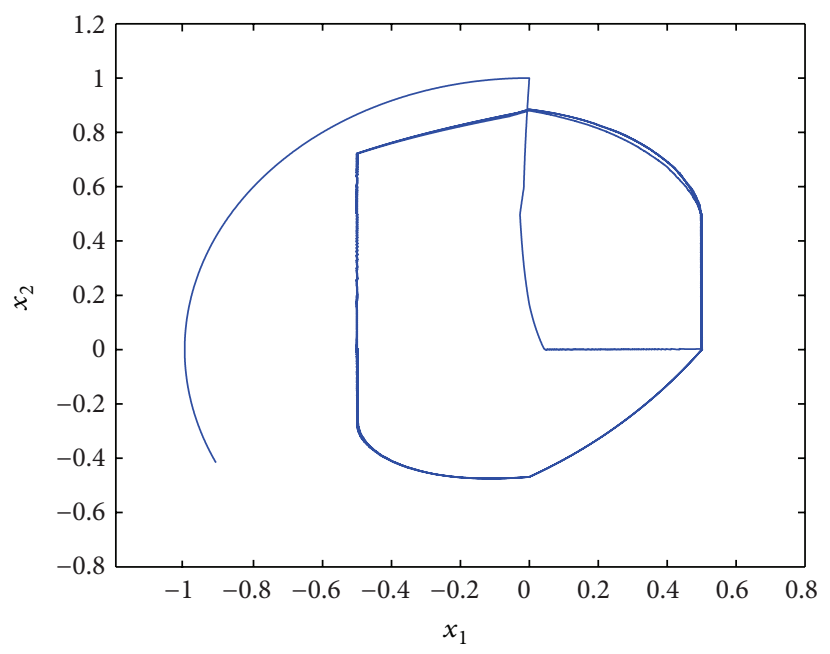

FIGURE 2: Phase plane behavior of the state variables $x_{1}$ and $x_{2}$ when $I(t)=(2 \sin t,-2 \cos t)^{T}$.

Figure 3 displays the state trajectories of this neural network with state initial value $\phi(t)=(\sin 2 t, \cos 2 t)^{T}, t \in$ $[-1,0]$, when $I(t)=(1,-3)^{T}$. It can be seen that these trajectories converge into the unique equilibrium point of the network. This is in accordance with the conclusion of Corollary 15.

Example 2. Consider the third-order neural network (4) with the following system parameters:

$$
\begin{gathered}
D=\left(\begin{array}{lll}
2 & 0 & 0 \\
0 & 2 & 0 \\
0 & 0 & 2
\end{array}\right), \quad A=\left(\begin{array}{ccc}
-3.00 & 0.53 & 0.13 \\
0.15 & -3.00 & 0.25 \\
0.30 & 0.25 & -3.00
\end{array}\right), \\
B=\left(\begin{array}{ccc}
0.80 & -0.65 & 0.06 \\
0.41 & -0.82 & 0.35 \\
0.27 & 0.34 & 0.60
\end{array}\right), \quad C=\left(\begin{array}{ccc}
-0.67 & 0.14 & 0.04 \\
-0.30 & 0.69 & 0.04 \\
0.05 & -0.25 & 0.75
\end{array}\right) .
\end{gathered}
$$




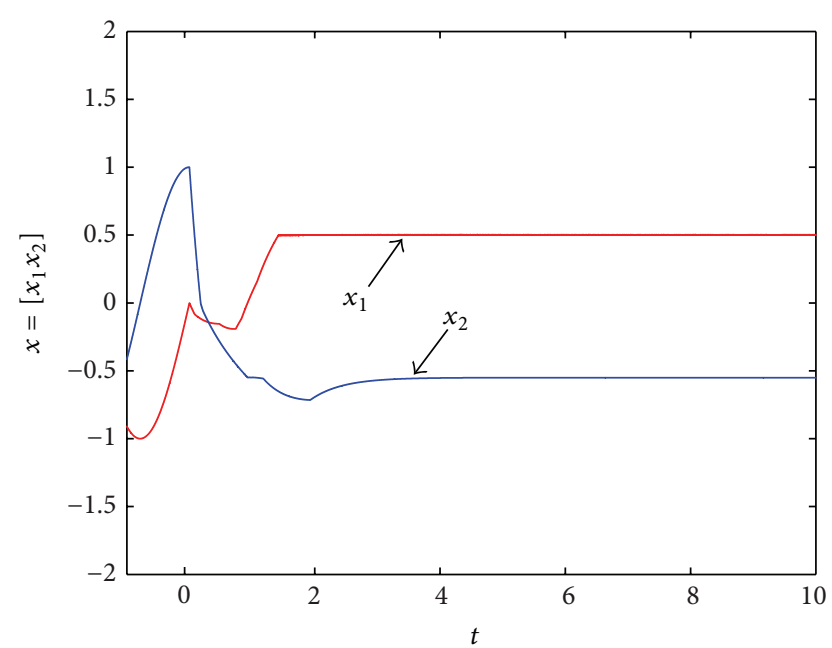

FIGURE 3: Time-domain behavior of the state variables $x_{1}$ and $x_{2}$ when $I(t)=(1,-3)^{T}$.

Set $g_{1}(s)=[s], g_{2}(s)=g_{3}(s)=[s+0.5], \tau(t)=0.8-0.2 \sin t$, and $\sigma(t)=0.5(1+\cos t)$. It is easy to check that assumptions $\left(A_{1}\right)-\left(A_{3}\right)$ hold, and $\tau_{M}=1, \tau_{D}=0.2, \sigma_{M}=1$, and $\sigma_{D}=0.5$.

By solving the LMI in (15) by using appropriate LMI solver in the Matlab, the feasible positive definite diagonal matrix $P$ and positive definite matrices $Q, H$ could be as

$$
\begin{gathered}
P=\left(\begin{array}{ccc}
22.8504 & 0 & 0 \\
0 & 23.5247 & 0 \\
0 & 0 & 23.3745
\end{array}\right), \\
Q=\left(\begin{array}{ccc}
41.7777 & -10.5914 & -0.2800 \\
-10.5914 & 43.1320 & -2.8559 \\
-0.2800 & -2.8559 & 41.0031
\end{array}\right), \\
H=\left(\begin{array}{ccc}
46.2777 & -11.2084 & -4.7838 \\
-11.2084 & 47.0601 & -3.6590 \\
-4.7838 & -3.6590 & 48.8991
\end{array}\right) .
\end{gathered}
$$

The assumption $\left(A_{4}\right)$ is also satisfied.

When the external input of the network $I(t)=(2 \sin t$, $-\cos t,-5 \sin 2 t)^{T}$ is a periodic function, by Corollary 14, this neural network has a unique periodic solution which is globally exponentially stable.

Figures 4 and 5 display the state trajectories of this neural network with state initial value $\phi(t)=\left(-(1 / 2) e^{2 t}\right.$, $\left.e^{t},-(1 / 5) \sin t\right)^{T}, t \in[-1,0]$, when $I(t)=(2 \sin t,-\cos t$, $-5 \sin 2 t)^{T}$. It can be seen that these trajectories converge into the unique periodic of the network. This is in accordance with the conclusion of Corollary 14.

When the external input of the network $I(t)=$ $(-3,-4,-5)^{T}$ is constant, by Corollary 15 , this neural network has a unique equilibrium point which is globally exponentially stable.

Figure 6 displays the state trajectories of this neural network with initial values $\phi(t)=\left(-(1 / 2) e^{2 t}, e^{t}\right.$, $-(1 / 5) \sin t)^{T}, t \in[-1,0]$, when $I(t)=(-3,-4,-5)^{T}$. It can be seen that these trajectories converge into the unique

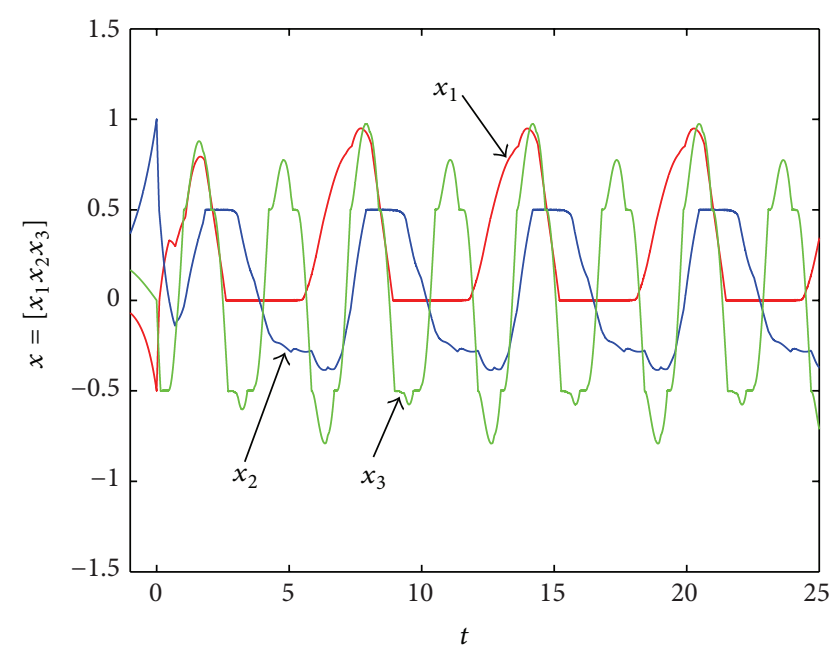

FIGURE 4: Time-domain behavior of the state variables $x_{1}, x_{2}$, and $x_{3}$ when $I(t)=(2 \sin t,-\cos t,-5 \sin 2 t)^{T}$.

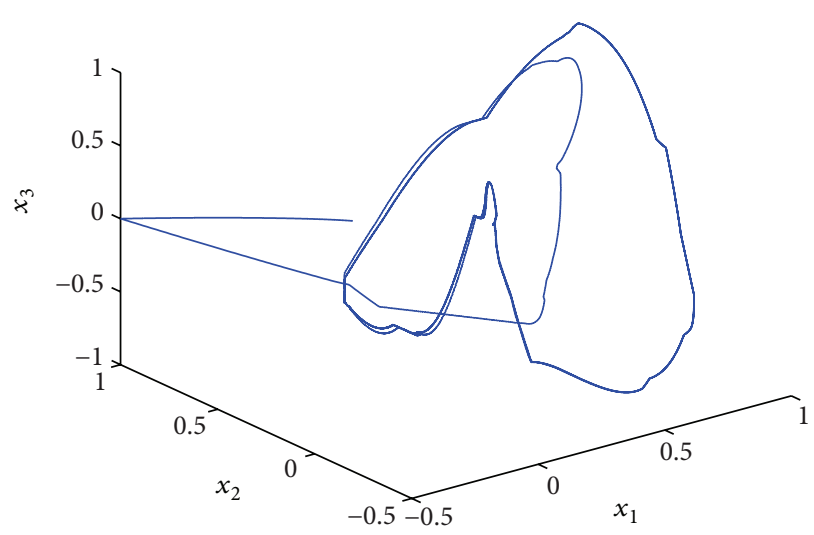

Figure 5: Phase plane behavior of the state variables $x_{1}, x_{2}$, and $x_{3}$ when $I(t)=(2 \sin t,-\cos t,-5 \sin 2 t)^{T}$.

equilibrium point of the network. This is in accordance with the conclusion of Corollary 15.

\section{Conclusion}

In this paper, the almost periodic oscillation issue has been investigated for the neural networks with mixed timevarying delays and discontinuous activation functions. Some sufficient conditions which ensure the existence, uniqueness, and global exponential stability of almost periodic solution have been obtained in terms of LMIs, which is easy to be checked and applied in practice. Two numerical examples have been given to illustrate the effectiveness of the present results.

In [2], Forti et al. conjectured that all solutions of the delayed neural networks with discontinuous neuron activations and periodic inputs converge into an asymptotically stable limit cycle. In this paper, under the assumptions 


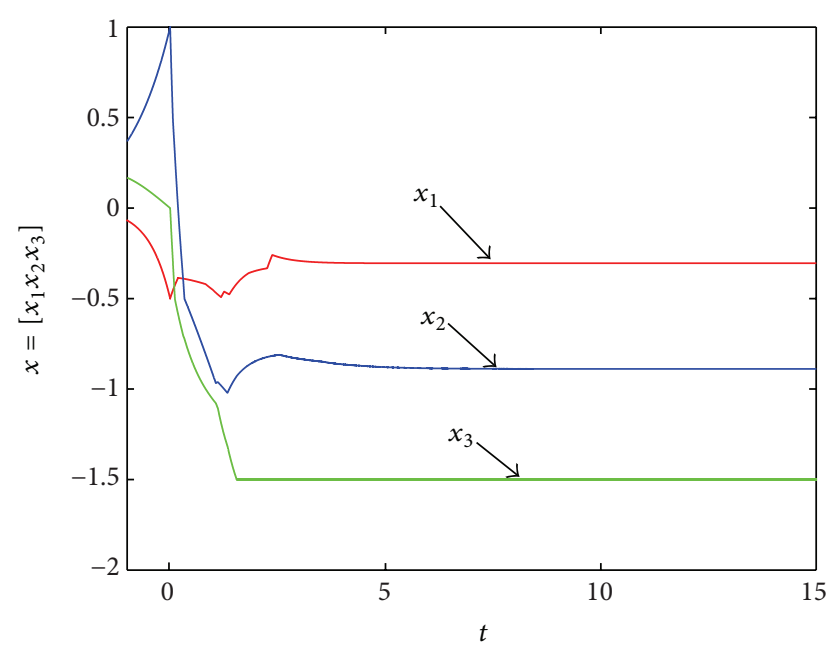

FIGURE 6: Time-domain behavior of the state variables $x_{1}, x_{2}$, and $x_{3}$ when $I(t)=(-3,-4,-5)^{T}$.

$\left(A_{1}\right)-\left(A_{4}\right)$, the obtained results confirm that Forti's conjecture is true for the neural networks with mixed timevarying delays and discontinuous activation functions. In the near future, the stability issues of almost periodic solution for stochastic/impulsive neural networks with discontinuous activations will be expected to be solved.

\section{Acknowledgments}

This work was supported by the National Science and Technology Major Project of China (2011ZX05020-006) and the Natural Science Foundation of Hebei Province of China (A2011203103).

\section{References}

[1] M. Forti and P. Nistri, "Global convergence of neural networks with discontinuous neuron activations," IEEE Transactions on Circuits and Systems I, vol. 50, no. 11, pp. 1421-1435, 2003.

[2] M. Forti, M. Grazzini, P. Nistri, and L. Pancioni, "Generalized lyapunov approach for convergence of neural networks with discontinuous or non-lipschitz activations," Physica D, vol. 214, no. 1, pp. 88-99, 2006.

[3] M. Forti, "M-matrices and global convergence of discontinuous neural networks," International Journal of Circuit Theory and Applications, vol. 35, no. 2, pp. 105-130, 2007.

[4] W. Lu and T. Chen, "Dynamical behaviors of Cohen-Grossberg neural networks with discontinuous activation functions," Neural Networks, vol. 18, no. 3, pp. 231-242, 2005.

[5] M. Forti, P. Nistri, and D. Papini, "Global exponential stability and global convergence in finite time of delayed neural networks with infinite gain," IEEE Transactions on Neural Networks, vol. 16, no. 6, pp. 1449-1463, 2005.

[6] L. Li and L. Huang, "Global asymptotic stability of delayed neural networks with discontinuous neuron activations," Neurocomputing, vol. 72, no. 16-18, pp. 3726-3733, 2009.

[7] S. Qin and X. Xue, "Global exponential stability and global convergence in finite time of neural networks with discontinuous activations," Neural Processing Letters, vol. 29, no. 3, pp. 189-204, 2009.

[8] Y. Meng, L. Huang, Z. Guo, and Q. Hu, "Stability analysis of Cohen-Grossberg neural networks with discontinuous neuron activations," Applied Mathematical Modelling, vol. 34, no. 2, pp. 358-365, 2010.

[9] L. Li and L. Huang, "Dynamical behaviors of a class of recurrent neural networks with discontinuous neuron activations," Applied Mathematical Modelling, vol. 33, no. 12, pp. 4326-4336, 2009.

[10] J. Liu, X. Liu, and W.-C. Xie, "Global convergence of neural networks with mixed time-varying delays and discontinuous neuron activations," Information Sciences, vol. 183, pp. 92-105, 2012.

[11] X. Nie and J. Cao, "Existence and global stability of equilibrium point for delayed competitive neural networks with discontinuous activation functions," International Journal of Systems Science, vol. 43, no. 3, pp. 459-474, 2012.

[12] Z. Guo and L. Huang, "LMI conditions for global robust stability of delayed neural networks with discontinuous neuron activations," Applied Mathematics and Computation, vol. 215, no. 3, pp. 889-900, 2009.

[13] X. Wu, Y. Wang, L. Huang, and Y. Zuo, "Robust exponential stability criterion for uncertain neural networks with discontinuous activation functions and time-varying delays," Neurocomputing, vol. 73, no. 7-9, pp. 1265-1271, 2010.

[14] X. Liu and J. Cao, "Robust state estimation for neural networks with discontinuous activations," IEEE Transactions on Systems, Man, and Cybernetics. Part B, vol. 40, no. 6, pp. 1425-1437, 2010.

[15] X. Liu, T. Chen, J. Cao, and W. Lu, "Dissipativity and quasisynchronization for neural networks with discontinuous activations and parameter mismatches," Neural Networks, vol. 24, no. 10, pp. 1013-1021, 2011.

[16] G. Bao and Z. Zeng, "Analysis and design of associative memories based on recurrent neural network with discontinuous activation functions," Neurocomputing, vol. 77, no. 1, pp. 101-107, 2012.

[17] X. Liu and J. Cao, "On periodic solutions of neural networks via differential inclusions," Neural Networks, vol. 22, no. 4, pp. 329-334, 2009.

[18] L. Huang, J. Wang, and X. Zhou, "Existence and global asymptotic stability of periodic solutions for hopfield neural networks with discontinuous activations," Nonlinear Analysis: Real World Applications, vol. 10, no. 3, pp. 1651-1661, 2009.

[19] H. Wu and Y. Li, "Existence and stability of periodic solution for BAM neural networks with discontinuous neuron activations," Computers Mathematics with Applications, vol. 56, no. 8, pp. 1981-1993, 2008.

[20] H. Wu, "Stability analysis for periodic solution of neural networks with discontinuous neuron activations," Nonlinear Analysis: Real World Applications, vol. 10, no. 3, pp. 1717-1729, 2009.

[21] H. Wu, "Global stability analysis of a general class of discontinuous neural networks with linear growth activation functions," Information Sciences, vol. 179, no. 19, pp. 3432-3441, 2009.

[22] H. Wu, X. Xue, and X. Zhong, "Stability analysis for neural networks with discontinuous neuron activations and impulses," International Journal of Innovative Computing, Information and Control, vol. 3, no. 6B, pp. 1537-1548, 2007.

[23] H. Wu and C. Shan, "Stability analysis for periodic solution of BAM neural networks with discontinuous neuron activations 
and impulses," Applied Mathematical Modelling, vol. 33, no. 6, pp. 2564-2574, 2009.

[24] D. Papini and V. Taddei, "Global exponential stability of the periodic solution of a delayed neural network with discontinuous activations," Physics Letters A, vol. 343, no. 1-3, pp. 117-128, 2005.

[25] X. Chen and Q. Song, "Global exponential stability of the periodic solution of delayed Cohen-Grossberg neural networks with discontinuous activations," Neurocomputing, vol. 73, no. 16-18, pp. 3097-3104, 2010.

[26] X. He, W. Lu, and T. Chen, "Nonnegative periodic dynamics of delayed Cohen-Grossberg neural networks with discontinuous activations," Neurocomputing, vol. 73, no. 13-15, pp. 2765-2772, 2010 .

[27] Z. Cai and L. Huang, "Existence and global asymptotic stability of periodic solution for discrete and distributed time-varying delayed neural networks with discontinuous activations," Neurocomputing, vol. 74, no. 17, pp. 3170-3179, 2011.

[28] Z. Cai, L. Huang, Z. Guo, and X. Chen, "On the periodic dynamics of a class of time-varying delayed neural networks via differential inclusions," Neural Networks, vol. 33, pp. 97-113, 2012.

[29] W. Allegretto, D. Papini, and M. Forti, "Common asymptotic behavior of solutions and almost periodicity for discontinuous, delayed, and impulsive neural networks," IEEE Transactions on Neural Networks, vol. 21, no. 7, pp. 1110-1125, 2010.

[30] W. Lu and T. Chen, "Almost periodic dynamics of a class of delayed neural networks with discontinuous activations," Neural Computation, vol. 20, no. 4, pp. 1065-1090, 2008.

[31] W. Ding and L. Wang, " $N^{2}$ almost periodic attractors for Cohen-Grossberg-type BAM neural networks with variable coefficients and distributed delays," Journal of Mathematical Analysis and Applications, vol. 373, no. 1, pp. 322-342, 2011.

[32] X. Huang and J. Cao, "Almost periodic solution of shunting inhibitory cellular neural networks with time-varying delay," Physics Letters A, vol. 314, no. 3, pp. 222-231, 2003.

[33] W. Lu and T. Chen, "Global exponential stability of almost periodic solution for a large class of delayed dynamical systems," Science in China Series A, vol. 48, no. 8, pp. 1015-1026, 2005.

[34] Y. Liu, Z. You, and L. Cao, "On the almost periodic solution of generalized hopfield neural networks with time-varying delays," Neurocomputing, vol. 69, no. 13-15, pp. 1760-1767, 2006.

[35] H. Xiang and J. Cao, "Almost periodic solution of CohenGrossberg neural networks with bounded and unbounded delays," Nonlinear Analysis: Real World Applications, vol. 10, no. 4, pp. 2407-2419, 2009.

[36] L. Wang, "Existence and global attractivity of almost periodic solutions for delayed high-ordered neural networks," Neurocomputing, vol. 73, no. 4-6, pp. 802-808, 2010.

[37] L. Wang, W. Lu, and T. Chen, "Multistability and new attraction basins of almost-periodic solutions of delayed neural networks," IEEE Transactions on Neural Networks, vol. 20, no. 10, pp. 15811593, 2009.

[38] C. Bai, "Existence and stability of almost periodic solutions of Hopfield neural networks with continuously distributed delays," Nonlinear Analysis: Theory, Methods \& Applications, vol. 71, no. 11, pp. 5850-5859, 2009.

[39] H. Jiang, L. Zhang, and Z. Teng, "Existence and global exponential stability of almost periodic solution for cellular neural networks with variable coefficients and time-varying delays," IEEE Transactions on Neural Networks, vol. 16, no. 6, pp. 13401351, 2005.
[40] S. Qin, X. Xue, and P. Wang, "Global exponential stability of almost periodic solution of delayed neural networks with discontinuous activations," Information Sciences, vol. 220, pp. 367-378, 2013.

[41] S. Boyd, L. E. Ghaoui, E. Feron, and V. Balakrishnan, Linear Matrix Inequalities in System and Control Theory, SIAM, Philadelphia, Pa, USA, 1994.

[42] F. H. Clarke, Oprimization and Non-Smooth Analysis, Wiley, New York, NY, USA, 1983.

[43] A. F. Filippov, Differential Equations with Discontinuous RightHand Side, Mathematics and Its Applications, Soviet Series, Kluwer Academic Publisher, Boston, Mass, USA, 1984.

[44] J.-P. Aubin and A. Cellina, Differential Inclusions, vol. 264, Springer, Berlin, Germany, 1984.

[45] B. M. Levitan and V. V. Zhikov, Almost Periodic Functions and Differential Equations, Cambridge University Press, Cambridge, UK, 1982. 


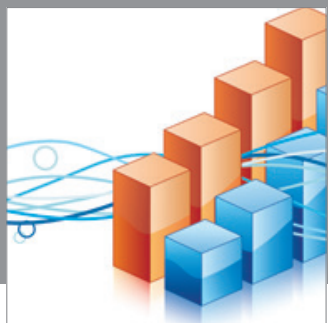

Advances in

Operations Research

mansans

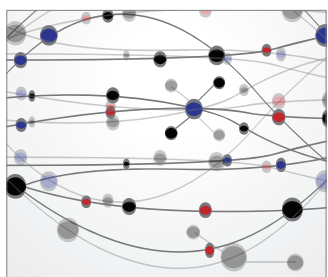

The Scientific World Journal
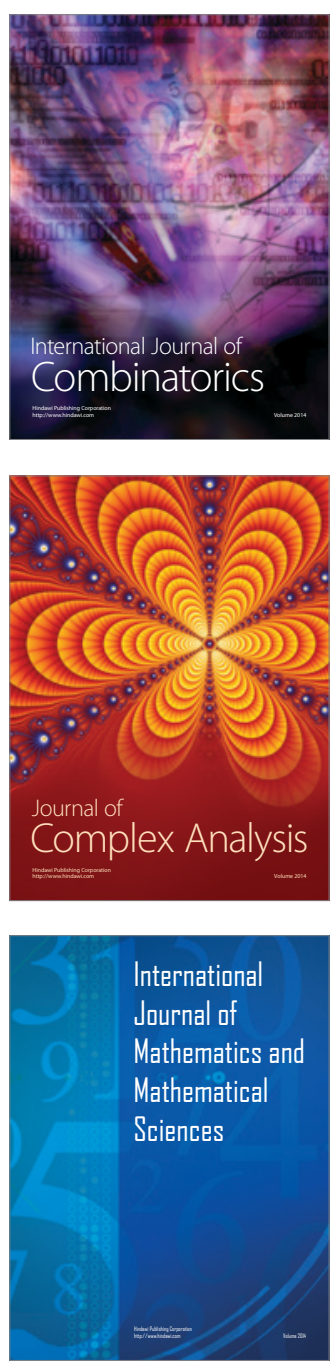
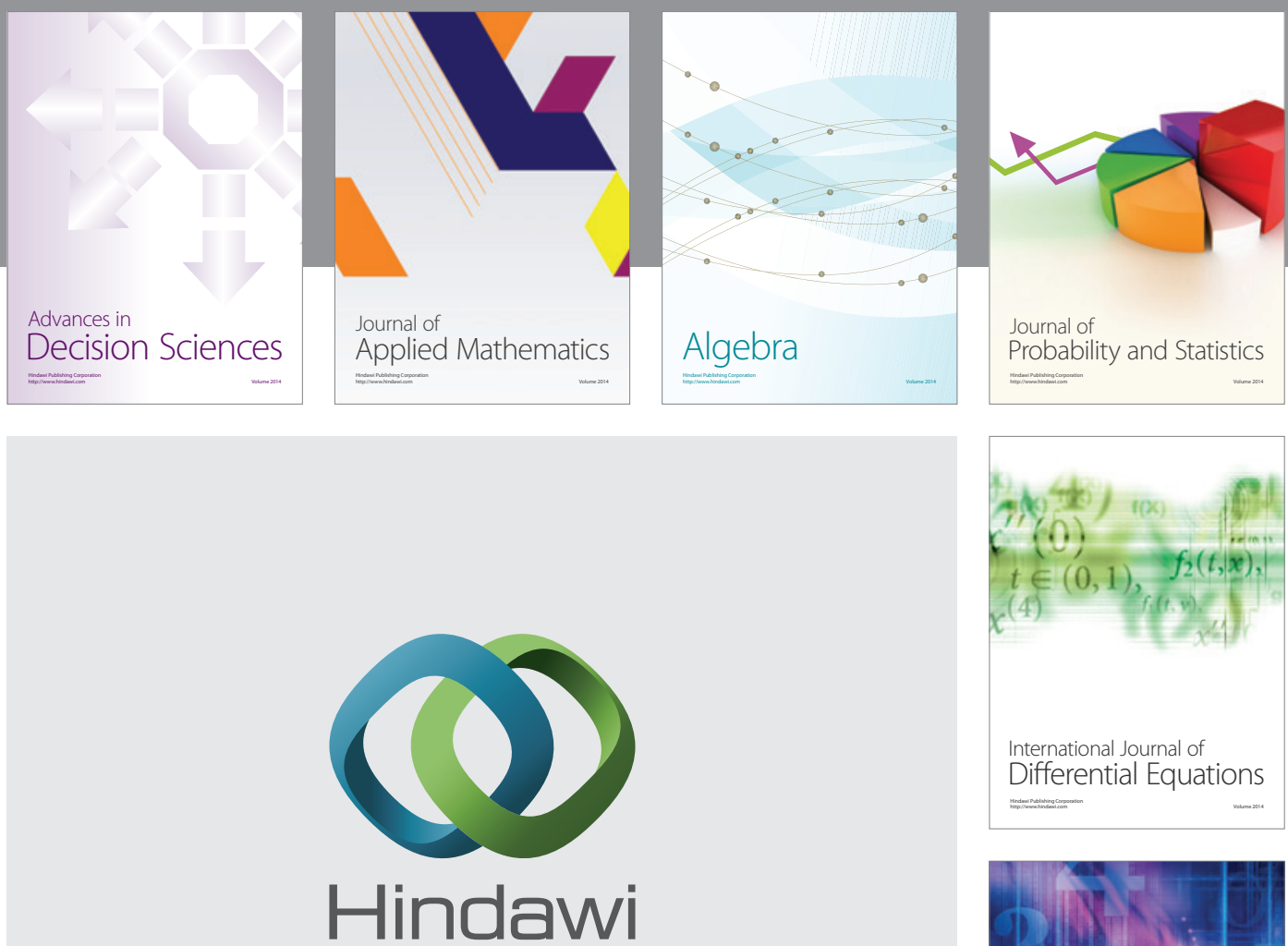

Submit your manuscripts at http://www.hindawi.com
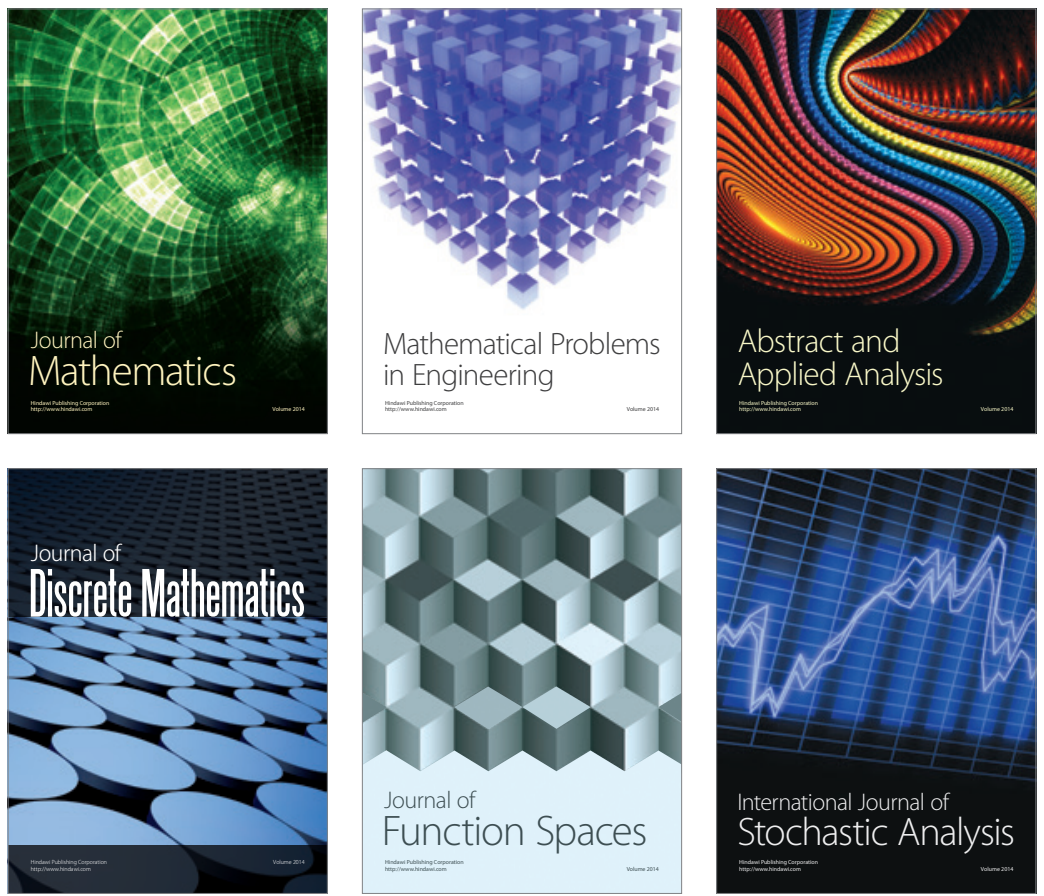

Journal of

Function Spaces

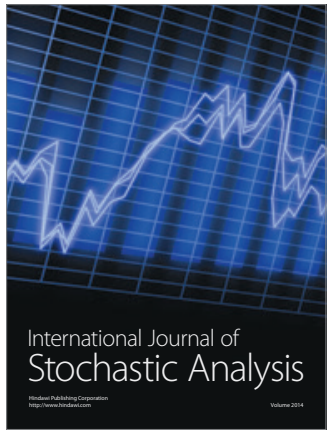

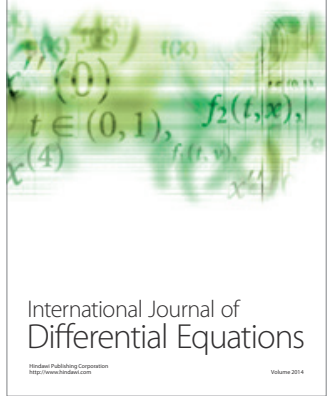
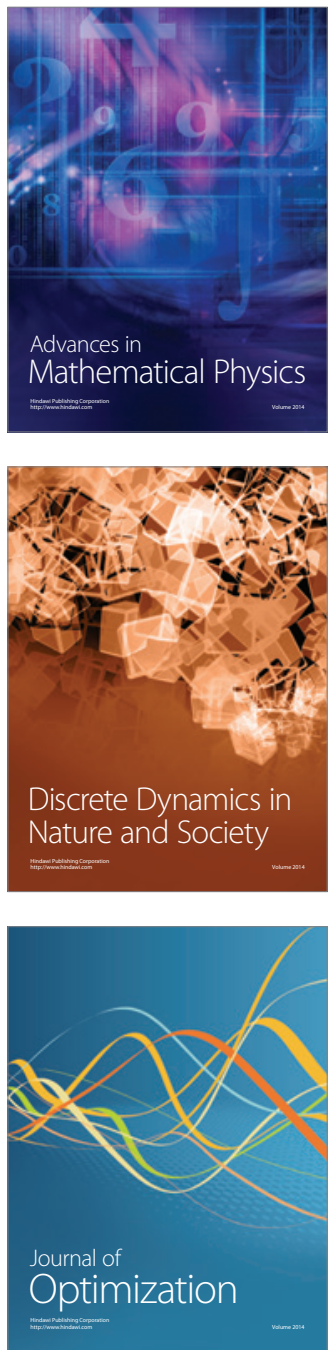